\title{
BERNSTEIN POLYNOMIALS FOR FUNCTIONS OF TWO VARIABLES OF CLASS $C^{(k)}$
}

\author{
EDWARD H. KINGSLEY ${ }^{1}$
}

Introduction. Let $\phi(x, y)$ be a continuous function of the real variables $x$ and $y$, where $x$ and $y$ are in the closed region $R: 0 \leqq x \leqq 1$, $0 \leqq y \leqq 1$. The Bernstein polynomial $B_{m n}(x, y)$ associated with the function $\phi(x, y)$ is defined as

$$
B_{m n}(x, y)=\sum_{p=0}^{n} \sum_{q=0}^{m} \phi\left(\frac{p}{n}, \frac{q}{m}\right) \lambda_{n, p}(x) \lambda_{m, q}(y)
$$

where $\lambda_{n, p}(x)=C_{n, p} x^{p}(1-x)^{n-p}, \lambda_{m, q}(y)=C_{m, q} y^{q}(1-y)^{m-q}$.

A function $\phi(x, y)$ is said to be of class $C^{(k)}$ for $x$ and $y$ in $R$, if the partial derivatives of order $1,2, \cdots, k$ of $\phi(x, y)$ exist and are continuous. We shall use the notation

$$
\phi^{(i, k-i)}=\frac{\partial^{(k)} \phi(x, y)}{\partial x^{i} \partial y^{k-i}} \quad(i=0,1, \cdots, k)
$$

and, for brevity, shall omit functional arguments from expressions whenever possible.

It is the purpose of this paper to prove the

Theorem. If $\phi(x, y)$ is of class $C^{(k)}$ for $x$ and $y$ in $R$, then

$$
\lim _{m, n=\infty} B_{m n}^{(i, k-i)}=\phi^{(i, k-i)}
$$

and the convergence is uniform in $R$.

This theorem, for functions of one variable and for $k=0$, was proved by $\mathrm{S}$. Bernstein [1], ${ }^{2}$ and again for functions of one variable but for arbitrary $k$, the theorem was proved by S. Wigert [2]. The process of extending the results of Bernstein and Wigert to functions of two variables of class $C^{(k)}$ introduces aspects which are of interest.

Preliminary results. We shall make use of the relations

$$
\sum_{p=0}^{n} \lambda_{n, p}(x)=1,
$$

Received by the editors December 12, 1949.

${ }^{1}$ This is part of a thesis written under the direction of Professor E. D. Hellinger for the degree M.S. at Northwestern University, June 1949.

${ }^{2}$ Numbers in brackets refer to the bibliography at the end of the paper. 
(3)

$$
\sum_{p=0}^{n}(n x-p)^{2} \lambda_{n, p}(x)=n x(1-x) \quad[3, \text { p. 152] }
$$

If we define, for $k \geqq 0, i=0,1, \cdots, k$,

$$
A_{p, q}^{(i, k-i)}=\sum_{\alpha=0}^{i} \sum_{\beta=0}^{k-i}(-1)^{\alpha+\beta} C_{i, \alpha} C_{k \rightarrow i, \beta} \phi\left(\frac{p+(i-\alpha)}{n}, \frac{q+(k-i-\beta)}{m}\right),
$$

then, by mathematical induction, the following two lemmas can be established.

LEMMA 1. If $0 \leqq i \leqq k, i \leqq n, k \leqq m, x$ and $y$ in $R$, then the $k$ th partial derivatives of the Bernstein polynomials (1) are given by

$$
B_{m n}^{(i, k-i)}=\frac{n ! m !}{(n-i) !(m-k+i) !} \sum_{p=0}^{n-i} \sum_{q=0}^{m-k+i} A_{p, q}^{(i, k-i)} \lambda_{n-i, p} \lambda_{m-k+i, q} .
$$

LEMMA 2. If $0 \leqq i \leqq k, 0 \leqq p \leqq n-i, 0 \leqq q \leqq m-k+i$, and if $\phi(x, y)$ is of class $C^{(k)}$ for $x$ and $y$ in $R$, then there exist two real numbers $\xi=\xi(p)$, $\gamma=\gamma(q)$ such that $0<\xi<1,0<\gamma<1$ and such that

$$
A_{p, q}^{(i, k-i)}=\frac{1}{n^{i} m^{k-i}} \phi^{(i, k-i)}\left(\frac{p+\xi i}{n}, \frac{q+\gamma(k-i)}{m}\right) .
$$

The next lemma is basic for the proof of the theorem.

Lemma 3. For fixed $x$ and $y$ in $R$ and for fixed positive integers $M$ and $N$, let $d$ be an arbitrary positive number and let $a(p, q)$ be a quantity dependent upon $p$ and $q$ and such that

$$
\begin{aligned}
& |a(p, q)| \leqq \pi_{1} \text { for }\left|x-\frac{p}{N}\right| \leqq d \text { and }\left|y-\frac{q}{M}\right| \leqq d, \\
& |a(p, q)| \leqq \pi_{2} \text { for }\left|x-\frac{p}{N}\right|>d \text { and }\left|y-\frac{q}{M}\right|>d .
\end{aligned}
$$

Furthermore, assume that it is possible to split off from $a(p, q)$ terms $a^{\prime}(p)$ independent of $q$ or terms $b^{\prime}(q)$ independent of $p$, that is,

$$
a(p, q)=a^{\prime \prime}(p, q)+a^{\prime}(p)=b^{\prime \prime}(p, q)+b^{\prime}(q)
$$

such that

$$
\begin{aligned}
& \left|a^{\prime \prime}(p)\right| \leqq \pi_{3} \text { for }\left|x-\frac{p}{N}\right| \leqq d \text { and }\left|y-\frac{q}{M}\right|>d \\
& \left|a^{\prime}(p, q)\right| \leqq \pi_{4} \text { for }\left|x-\frac{p}{N}\right| \leqq d
\end{aligned}
$$


(8)

$$
\begin{aligned}
& \left|b^{\prime \prime}(p, q)\right| \leqq \pi_{6} \text { for }\left|x-\frac{p}{N}\right|>d \text { and }\left|y-\frac{q}{M}\right| \leqq d \\
& \left|b^{\prime}(q)\right| \leqq \pi_{0} \text { for }\left|y-\frac{q}{M}\right| \leqq d
\end{aligned}
$$

then

$$
\begin{aligned}
\left|\sum_{p=0}^{N} \sum_{q=0}^{M} a(p, q) \lambda_{N, p} \lambda_{M, q}\right| & \\
& \leqq \pi_{1}+\pi_{4}+\pi_{6}+\frac{\pi_{2}(M+N)}{8 M N d^{2}}+\frac{\pi_{3}}{4 M d^{2}}+\frac{\pi_{6}}{4 N d^{2}} .
\end{aligned}
$$

Proof. Consider the inequality

$$
\begin{aligned}
\left|\sum_{p=0}^{N} \sum_{q=0}^{M} a(p, q) \lambda_{N, p} \lambda_{M, q}\right| & \\
& \leqq \sum_{|x-p / N| \leqq d} \sum_{|y-q / M| \leqq d}|a(p, q)| \lambda_{N, p} \lambda_{M, q} \\
& +\sum_{|x-p / N|>d} \sum_{|y-q / M|>d}|a(p, q)| \lambda_{N, p} \lambda_{M, q} \\
& +\sum_{|x-p / N| \leqq d} \sum_{|y-q / M|>d}\left|a^{\prime \prime}(p, q)+a^{\prime}(p)\right| \lambda_{N, p}+\lambda_{M, q} \\
& +\sum_{|x-p / N|>d} \sum_{|y-q / M| \leqq d}\left|b^{\prime \prime}(p, q)+b^{\prime}(q)\right| \lambda_{N, p} \lambda_{M, q} \\
& =s_{1}+s_{2}+s_{3}+s_{4},
\end{aligned}
$$

then

$$
\begin{aligned}
s_{1} \leqq \pi_{1} & \sum_{|x-p / N| \leqq d} \sum_{|y-q / M| \leqq d} \lambda_{N, p} \lambda_{M, q} \\
& \leqq \pi_{1} \sum_{p=0}^{N} \sum_{q=0}^{M} \lambda_{N, p} \lambda_{M, q}=\pi_{1}
\end{aligned}
$$

and hence $s_{1} \leqq \pi_{1}$.

The inequalities in (5) imply the inequality

$$
\frac{M^{2}(M x-p)^{2}+N^{2}(M y-q)^{2}}{2 M^{2} N^{2} d^{2}}>1
$$

and by (5) again

$$
|a(p, q)| \leqq \pi_{2}<\frac{\pi_{2}}{2 M^{2} N^{2} d^{2}}\left\{M^{2}(N x-p)^{2}+N^{2}(M y-q)^{2}\right\},
$$


thus

$$
\begin{array}{rlr}
s_{2} & \leqq \frac{\pi_{2}}{2 M^{2} N^{2} d^{2}} \sum_{p=0}^{N} \sum_{q=0}^{M}\left\{M^{2}(N x-p)^{2}+N^{2}(M y-q)^{2}\right\} \lambda_{N, p} \lambda_{M, Q} \\
& =\frac{\pi_{2}}{2 M N d^{2}}[M x(1-x)+N y(1-y)] \quad \text { (by (3) and }
\end{array}
$$

and since

$$
\max _{x, y \in R}[M x(1-x)+N y(1-y)]=\frac{M+N}{4},
$$

we have

$$
s_{2} \leqq \frac{\pi_{2}(M+N)}{8 M N d^{2}} .
$$

In similar fashion, using (6), (7), (8), and (9), we obtain

$$
s_{3} \leqq \pi_{4}+\frac{\pi_{3}}{4 M d^{2}}, \quad s_{4} \leqq \pi_{6}+\frac{\pi_{5}}{4 N d^{2}}
$$

Collecting these estimates in (11) we obtain (10).

Proof of the theorem. By Lemmas 1 and 2, we have the inequality

$$
\begin{aligned}
\left|B_{m n}^{(i, k-i)}-\phi^{(i, k-i)}\right| & \leqq \mid \sum_{p=0}^{n-i} \sum_{q=0}^{m-k+i}\left\{1-\frac{n ! m !}{(n-i) !(m-k+i) ! n^{i} m^{k-i}}\right\} \\
& \cdot \phi^{(i, k-i)}\left(\frac{p+\xi i}{n}, \frac{q+\gamma(k-i)}{m}\right) \lambda_{n-i, p} \lambda_{m-k+i, q} \mid \\
& +\mid \sum_{p=0}^{n-i} \sum_{q=0}^{m-k+i}\left\{\phi^{(i, k-i)}\left(\frac{p+\xi i}{n}, \frac{q+k(k-i)}{m}\right)\right. \\
& \left.-\phi^{(i, k-i)}(x, y)\right\} \lambda_{n-i, p} \lambda_{m-k+i, q} \mid=S_{1}+S_{2 .}
\end{aligned}
$$

We first estimate $S_{2}$. Let $\phi^{(i, k-i)}(x, y)=\psi(x, y)$, then since $\psi(x, y)$ is continuous for $x$ and $y$ in $R$, it is uniformly continuous and bounded on $R$. That is, for an arbitrary positive number $\epsilon$, there exists a number $d(\epsilon)>0$ such that if $\left|x-x_{1}\right| \leqq d(\epsilon) / 2$ and $\left|y-y_{1}\right|$ $\leqq d(\epsilon) / 2$, where $x_{1}$ and $y_{1}$ are in $R$, then

$$
\left|\psi\left(x_{1}, y_{1}\right)-\psi(x, y)\right| \leqq \epsilon / 6
$$


and

$$
|\psi(x, y)| \leqq L^{(k)}, \quad \text { for } x \text { and } y \text { in } R,
$$

where $L^{(k)}=\max x, y \in R|\psi(x, y)|$.

If $p, n, i$ are positive integers such that $0 \leqq p \leqq n-i$, and if $\xi$ is a real number such that $0<\xi<1$, then

$$
\left|\frac{p}{n-i}-\frac{p+\xi i}{n}\right|<\frac{i}{n},
$$

and if $m, q, k, i$ are positive integers such that $0 \leqq q \leqq m-(k-i)$, and $\gamma$ a real number such that $0<\gamma<1$, then

$$
\left|\frac{q}{m-k+i}-\frac{q+\gamma(k-i)}{m}\right|<\frac{k-i}{m} .
$$

Now if $x$ is fixed and $p$ is such that $0 \leqq p \leqq n-1$ and $|x-p /(n-i)|$ $\leqq d(\epsilon) / 2$, then by $(14)$

$$
\left|x-\frac{p+\xi i}{n}\right| \leqq \frac{d(\epsilon)}{2}+\frac{i}{n}
$$

and if we choose

$$
N_{1 \epsilon}>2 i / d(\epsilon)+i
$$

then for $n>N_{16}$

$$
i / n<d(\epsilon) / 2
$$

and

$$
\left|x-\frac{p+\xi i}{n}\right| \leqq d(\epsilon) \quad \text { if } n>N_{1 \epsilon} .
$$

In the same manner, choose

$$
M_{1 \epsilon}>(k-i)+\frac{2(k-i)}{d(\epsilon)} ;
$$

then for fixed $y$ and $q$ such that $0 \leqq q \leqq m-k+i$ and $|y-q /(m-k+i)|$ $\leqq d(\epsilon) / 2$, we obtain from (15)

$$
\left|y-\frac{q+\gamma(k-i)}{m}\right| \leqq d(\epsilon) \quad \text { if } m>M_{1 \epsilon} .
$$

If we choose $x_{1}=(p+\xi i) / n, y_{1}=(q+\gamma(k-i)) / m$ in (12), then from (16), (17) we have 


$$
\left|\psi\left(\frac{p+\xi i}{n}, \frac{q+\gamma(k-i)}{m}\right)-\psi(x, y)\right| \leqq \frac{\epsilon}{6}
$$

if $n>N_{1 \epsilon}, \quad m>M_{1 \epsilon},|x-(p+\xi i) / n| \leqq d(\epsilon) / 2,|y-(q+\gamma(k-i)) / m|$ $\leqq d(\epsilon) / 2$.

If we now let:

$\Delta_{n-i}$ indicate summation for all $p$ such that $|x-p /(n-i)| \leqq d(\epsilon) / 2$, $\Delta_{n-i}^{\prime}$ indicate summation for all $p$ such that $|x-p /(n-i)|>d(\epsilon) / 2$, $\Delta_{m-k+i}$ indicate summation for all $q$ such that $|y-q /(m-k+i)|$ $\leqq d(\epsilon) / 2$,

$\Delta_{m-k+1}^{\prime}$ indicate summation for all $q$ such that $|y-q /(m-k+i)|$ $>d(\epsilon) / 2$,

then $S_{2}$ may be written as

$$
\begin{aligned}
& S_{2} \leqq \sum_{\Delta_{n-i}} \sum_{\Delta_{m-k+i}} \mid \psi\left(\frac{p+\xi i}{n}, \frac{q+\gamma(k-i)}{m}\right) \\
& -\psi(x, y) \mid \lambda_{n-i, p} \lambda_{m-k+i, q} \\
& +\sum_{\Delta_{m-i}^{\prime}} \sum_{\Delta_{\dot{m}-k+i}} \mid \psi\left(\frac{p+\xi i}{n}, \frac{q+\gamma(k-i)}{m}\right) \\
& -\psi(x, y) \mid \lambda_{n-i, p} \lambda_{m-k+i, q} \\
& +\sum_{\Delta_{n-i}} \sum_{\Delta_{m-k+i}} \mid \psi\left(\frac{p+\xi i}{n}, \frac{q+\gamma(k-i)}{m}\right) \\
& -\psi\left(\frac{p+\xi i}{n}, y\right) \mid \lambda_{n-i, p} \lambda_{m-k+i, q} \\
& +\sum_{\Delta_{n-i}} \sum_{\Delta_{m=k+i}^{\prime}}\left|\psi\left(\frac{p+\xi i}{n}, y\right)-\psi(x, y)\right| \lambda_{n-i, p} \lambda_{m-k+i, q} \\
& +\sum_{\Delta_{n}-i} \sum_{\Delta_{m-k+i}} \mid \psi\left(\frac{p+\xi i}{n}, \frac{q+\gamma(k-i)}{m}\right) \\
& -\psi\left(x, \frac{q+\gamma(k-i)}{m}\right) \mid \lambda_{m-i, p} \lambda_{m-k+i, q} \\
& +\sum_{\Delta_{i-i}} \sum_{\Delta_{m-k+i}} \mid \psi\left(x, \frac{q+\gamma(k-i)}{m}\right) \\
& -\psi(x, y) \mid \lambda_{n-i, p} \lambda_{m-k+i, q}
\end{aligned}
$$


If we use (13) and (18) and let

$$
\begin{aligned}
a(p, q) & =\psi\left(\frac{p+\xi i}{n}, \frac{q+\gamma(k-i)}{m}\right)-\psi(x, y), \\
d & =d(\epsilon) / 2, \\
\pi_{1} & =\pi_{1}=\pi_{0}=\epsilon / 6, \\
\pi_{2} & =\pi_{3}=\pi_{5}=2 L^{(k)},
\end{aligned}
$$

then the hypotheses of Lemma 3 are satisfied and (19) becomes

$$
S_{2} \leqq \frac{\epsilon}{2}+\frac{3 L^{(k)}}{[d(\epsilon)]^{2}}\left(\frac{1}{n-i}+\frac{1}{m-k+i}\right), \text { for } n>N_{1 \epsilon}, m>M_{1 \epsilon} \text {. }
$$

To estimate $S_{1}$, we note that there exist two numbers $N_{2 \epsilon}>i, M_{2 \epsilon}$ $>k-i$ such that if $n>N_{2 e}, m>M_{2 \epsilon}$, then

$$
\left|1-\frac{n ! m !}{(n-i) !(m-k+i) ! n^{i} m^{k-i}}\right|<\frac{\epsilon}{6 L^{(k)}} .
$$

Thus

$$
\begin{array}{rlr}
S_{1} & \leqq \frac{\epsilon}{6 L^{(k)}} \sum_{p=0}^{n-i} \sum_{q=0}^{m-k+i}\left|\psi\left(\frac{p+\xi i}{n}, \frac{q+\gamma(k-i)}{m}\right)\right| \lambda_{n-i, p} \lambda_{m-k+i, q} \\
& \leqq \frac{\epsilon}{6} \sum_{p=0}^{n-i} \sum_{q=0}^{m-k+i} \lambda_{n-i, p} \lambda_{m-k+i, q} & \text { by }
\end{array}
$$

and hence

$$
S_{1} \leqq \epsilon / 6 \text {. }
$$

Using these estimates of $S_{1}$ and $S_{2}$ we have

$$
\left|B_{m n}^{(i, k-i)}-\phi^{(i, k-i)}\right| \leqq \frac{2}{3} \epsilon+\frac{3 L^{(k)}}{[d(\epsilon)]^{2}}\left[\frac{1}{n-i}+\frac{1}{m-k+i}\right]
$$

for $n>N_{1 e,} N_{2 \varepsilon} ; m>M_{1 e}, M_{2 \epsilon}$.

Let

$$
\begin{aligned}
& N_{3 e}>i+\frac{18 L^{(k)}}{\epsilon[d(\epsilon)]^{2}}, \\
& M_{3 \epsilon}>(k-i)+\frac{18 L^{(k)}}{\epsilon[d(\epsilon)]^{2}},
\end{aligned}
$$

and take 


$$
\begin{aligned}
& N_{\mathrm{e}}=\max \left(N_{1 \mathrm{e}}, N_{2 \epsilon}, N_{\mathrm{se}}\right), \\
& M_{\mathrm{e}}=\max \left(M_{1 \mathrm{e}}, M_{2 e}, M_{8 \epsilon}\right) .
\end{aligned}
$$

In (21) let $n>N$, and such that

$$
\frac{3 L^{(k)}}{[d(\epsilon)]^{2}(n-i)}<\frac{\epsilon}{6}
$$

and in (22) let $m>M_{6}$ and such that

$$
\frac{3 L^{(k)}}{[d(\epsilon)]^{2}(m-k+i)}<\frac{\epsilon}{6} .
$$

Thus, for $n>N_{\text {c }}$ and $m>M_{\mathrm{e}}$ (20) becomes

$$
\left|B_{m n}^{(i, k-i)}-\phi^{(i, k-i)}\right|<\epsilon
$$

and the theorem is proved.

\section{BIBLIOGRAPHY}

1. S. Bernstein, Soc. Math. Charkow (2) vol. 13 (1912-1913).

2. S. Wigert, Sur l'approximation par polynomos, dés fonctions continues, Arkiv forr Mathematik, Astronomi och Fysik vol. 22B (1932) pp. 1-4.

3. D. V. Widder, The Laplace transform, Princeton, 1946.

Roosevelt College 